\title{
Patient considerations in the management of toe onychomycosis - role of efinaconazole
}

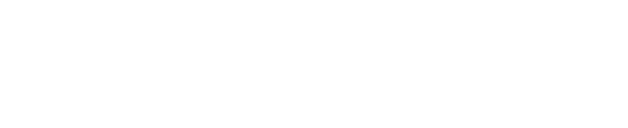

\section{Charlotte E LaSenna Antonella Tosti}

Department of Dermatology and Cutaneous Surgery, Miller School of Medicine, University of Miami, Miami, FL, USA
Correspondence: Charlotte LaSenna Department of Dermatology and Cutaneous Surgery, Miller School of Medicine, University of Miami, 1600 Northwest, I0th Avenue, RMSB Building, Room 2023C, Miami, FL 33। 36, USA

Tel +l 3052435523

Fax +I 3052435810

Email celasenna@med.miami.edu

\begin{abstract}
Onychomycosis is a difficult diagnosis to manage and treatment is sometimes avoided, as this diagnosis is often wrongly perceived as a cosmetic problem. However, onychomycosis has a negative impact on patients' quality of life, affecting social interaction, psychological well-being, and physical activities. Onychomycosis is also a risk factor for patients with diabetes, with proven increased rates of cellulitis, gangrene, and foot ulcers. Treatments are only mild to moderately effective, and rates of relapse and reinfection are high. Oral treatments require laboratory monitoring due to risk of hepatotoxicity and may be contraindicated in some patients due to risk of drug-drug interactions. Topical treatments require prolonged application and are not very effective. Efinaconazole $10 \%$ solution is a new topical triazole treatment for mild to moderate distal subungual onychomycosis, with good efficacy and without the need for debridement of nails. In onychomycosis of the toenails, efinaconazole $10 \%$ solution is documented to have a statistically significant, positive impact on patient satisfaction and quality of life.
\end{abstract}

Keywords: nail, fungus, quality of life, treatment, antifungal, dermatophyte

\section{Introduction}

Onychomycosis describes a common infection of the nail unit by dermatophytes, yeasts, or nondermatophytic molds that affects the toenails more frequently than fingernails. ${ }^{1}$ The negative impact on patients is more than cosmetic. Although this is often wrongly perceived as a superficial problem, there can be a significant impact on quality of life (QOL). ${ }^{2,3}$ When compared to healthy individuals, persons with onychomycosis had significantly lower ratings on assessment of self-perception of general health, bodily pain, mental health, social functioning, health concern, physical appearance, and functional limitations in activities on foot. ${ }^{3}$ Sequela of the disease include pain and discomfort, ${ }^{4}$ spread to other nails in the same patient or to the skin and nails of other family members, ${ }^{4-6}$ and increased risk of bacterial infections, such as cellulitis, particularly in patients with diabetes. ${ }^{7,8}$ Patients with diabetes and onychomycosis are at a three-fold higher risk of developing complications such as foot ulcer and gangrene compared to diabetic patients without onychomycosis. ${ }^{9}$ Nearly $90 \%$ of patients desire treatment, and $41 \%$ are even willing to accept treatment-associated side effects in order to be treated. ${ }^{3}$

Many nail dystrophies may mimic onychomycosis clinically, so it is important that a definitive diagnosis be established to avoid unnecessary costs or complications of treatment. ${ }^{10}$ Treatment of onychomycosis should only be performed after clinical assessment and microbiologic confirmation of the presence of hyphae or spores via $\mathrm{KOH}$ examination of scrapings of the nail bed, fungal culture, polymerase chain reaction, and/or microscopic examination, which may include periodic acid-Schiff stain of a nail clipping. ${ }^{11,12}$ 
Onychomycosis of the toenails is difficult to treat, and recurrence rate after cure is high. ${ }^{13}$ Systemic therapies are more effective than topical therapies, but their use can be limited by risk of drug interactions and systemic side effects. Patients often prefer and request topical treatments; however, penetration of the nail plate is difficult and the overall efficacy is lower than with systemic therapy.

The US Food and Drug Administration (FDA) recently approved efinaconazole, a topical antifungal, for the treatment of mild to moderate distal subungual onychomycosis (DLSO) of the toenails, defined as 20\%-50\% involvement of the target toenail. ${ }^{14}$ The objective of this article is to review onychomycosis from a patient-focused viewpoint and to discuss the efficacy and safety of efinaconazole.

\section{Onychomycosis basics}

Dermatophyte fungi are the most common culprits in onychomycosis, with Trichophyton rubrum and Trichophyton mentagrophytes species isolated in $80 \%-90 \%$ of cases. ${ }^{15,16}$ However, nondermatophyte fungi such as Scopulariopsis brevicaulis, Fusarium spp., and Aspergillus spp. can also contribute, especially in Europe and South America. ${ }^{17}$ Tinea pedis is a common dermatophyte infection of the foot that is often associated with onychomycosis, ${ }^{18}$ where the dermatophyte causing tinea pedis is thought to spread from the sole to the nails. ${ }^{19}$

There are several subtypes of onychomycosis. ${ }^{20-22}$ DLSO is the most common type, usually due to T. rubrum, and most commonly involves the first toe. Fungi reach the nail bed through the hyponychium causing subungual hyperkeratosis and onycholysis; proximal spreading often occurs with yellow-white streaks. The nail plate shows yellow-white discoloration. Proximal subungual onychomycosis (PSO) is much less common and starts proximally at the cuticle and moves distally. PSO due to T. rubrum may be a marker of severe immune suppression, such as AIDS..$^{23,24}$ White superficial onychomycosis is most commonly due to T. mentagrophytes and is an infection of the superficial nail plate. It appears as dull white spots on the nail plate surface that spread centrifugally and may involve one or several nails. The white areas easily scrape off with light curettage.

\section{Limitations of therapy}

Treatment options for onychomycosis are limited in number and efficacy. Relapse due to incomplete treatment and reinfection can occur in up to $25 \%$ of patients, ${ }^{13}$ with many suffering a long history of disease recurrence. ${ }^{25}$ In the United States, the only approved treatments for onychomycosis include topical ciclopirox, and systemic terbinafine and itraconazole. Topical amorolfine is available in Europe and South America but not in the United States. Systemic antifungals are more effective than topical antifungals, but their use can be limited by possible drug-drug interactions (DDIs) and side effects. ${ }^{26}$ Topical ciclopirox has limited efficacy and requires a long treatment period of up to 48 weeks of daily application and debridement. Some authors suggest to combine oral antifungals with topical treatment and nail debridement ${ }^{27}$ or avulsion ${ }^{28}$ to decrease duration of therapy and likelihood of adverse effects. Sometimes, physicians discourage patients from undergoing treatment, despite patient desire, as they are concerned of possible side effects, limited efficacy, and frequent relapse. ${ }^{3}$

Choice of treatment regimen should be based on patient health, associated disease, and severity of onychomycosis. Topical therapy is appropriate for mild to moderate DLSO and as initial treatment of classic white superficial onychomycosis. Some doctors also prescribe topical therapy to patients who have already reached clearance with oral therapy in order to prevent reinfection..$^{29}$ It may also be combined with systemic treatment in order to increase success rate.

\section{Barriers to the use of systemic therapies}

Approved systemic therapies include oral terbinafine and oral itraconazole. Oral fluconazole, although effective, is not approved for this indication in the United States. ${ }^{30-33}$ Most dermatologists consider terbinafine $250 \mathrm{mg}$ daily for 3 months the "gold standard" treatment for onychomycosis. ${ }^{34}$ Terbinafine is a safe medication, and side effects are uncommon, but cases of severe liver toxicity have been reported. Itraconazole is less frequently utilized as it is less effective than terbinafine ${ }^{35}$ and has more drug interactions. ${ }^{15}$ The approved dose for toenail onychomycosis is $200 \mathrm{mg}$ daily for 3 months, but several studies show that an intermittent regimen of $400 \mathrm{mg}$ daily for 1 week a month is also effective. ${ }^{35,36}$ Fluconazole is utilized off-label as pulse therapy at a dosage of 150 or $300 \mathrm{mg}$ once a week for several months (until cure). ${ }^{37}$ This pulse regimen makes drug interactions and hepatotoxicity less common.

Terbinafine and itraconazole are not recommended for patients with active or chronic liver disease. ${ }^{15}$ Monitoring of hepatic function is recommended prior to and during treatment for all patients, but especially in patients with underlying liver disease. ${ }^{15,34}$ Thus, systemic therapies are not a good option for patients who are unwilling or unable to have routine follow-up.

Additionally, DDIs may occur with systemic therapies because itraconazole and fluconazole inhibit CYP3A4 and 
terbinafine is an inhibitor of CYP2D6. ${ }^{15}$ Therefore they may cause alterations in metabolism of other drugs. Upon review of the patient's medication list, the physician may not be able to prescribe a systemic therapy due to known DDIs.

Cost of therapy with oral antifungals has considerably decreased after patent expiration. The last cost analysis of onychomycosis (including cost of medication, medical management, and management of adverse reactions) was performed in 1999, and a summary of the values to achieve mycologic cure in each patient is summarized as follows: griseofulvin US\$4,917, itraconazole (continuous therapy) US\$2,072, itraconazole (pulse therapy) US\$1,072, terbinafine US\$1,042, and fluconazole US\$1,449. ${ }^{38}$ The estimated medication cost for complete cure using ciclopirox lacquer is between US\$1,381 and US\$2,135; 39 however, with other medical and management costs, the estimated cost of complete cure increased to US\$17,029-US\$26,317.40 The cost for complete cure using efinaconazole has not yet been estimated. However, it should be kept in mind that topical therapies for onychomycosis are often not covered by medical insurance. On a larger scale, approximately US\$43 million (1997 values) is spent per year on the management of onychomycosis to cover 13 million visits by 662,000 patients over the age of $65 .^{41}$

Patient compliance is a barrier to treatment of onychomycosis because prolonged treatment is necessary as nail growth is a slow process. Problems are more common in children, the elderly, and immunocompromised patients. ${ }^{42,43}$ Important determinants of compliance with oral antifungal medications include: duration of therapy, ease of swallowing, frequency of dosage, and number of pills per intake. ${ }^{44}$ Overall compliance with oral medications (intermittent pulse itraconazole, intermittent terbinafine, continuous terbinafine) for the treatment of onychomycosis was found to be $45 \%$ due to adverse effects $(30 \%)$, discontinuation after perceived progress $(22 \%)$, and financial barriers (16\%). ${ }^{45}$ Another systematic review and meta-analysis found that $19 \%$ of patients discontinued oral itraconazole due to an adverse reaction and $1.5 \%$ discontinued due to hepatotoxicity. ${ }^{46}$

\section{Efficacy comparison}

Phase III studies reported complete cure rates of $14 \%$ with itraconazole and $38 \%$ with terbinafine and mycologic cure rates of $54 \%$ and $70 \%$, respectively (Sporanox package insert; Janssen Pharmaceuticals, Inc., NJ, USA: Lamisil package insert; Novartis, Basel, Switzerland). Subsequently, mycological cure rates were studied in a meta-analysis and are as follows: terbinafine $76 \% \pm 3 \%$, itraconazole pulse therapy $63 \% \pm 7 \%$, griseofulvin $60 \% \pm 6 \%$, itraconazole continuous therapy
$59 \% \pm 5 \%$, and fluconazole $48 \% \pm 5 \% .{ }^{47}$ Reported clinical cure rates are similar to mean mycological cure rates but are harder to assess across trials as they often have varying definitions of cure and different follow-up periods. ${ }^{47}$ Randomized clinical trials for ciclopirox lacquer have shown a complete cure rate of $5.5 \%-8.5 \%$ and a mycologic cure rate of $34 \% .{ }^{48}$

Efinaconazole $10 \%$ solution, a topical triazole antifungal solution, is a new option for topical treatment of mild to moderate DLSO. ${ }^{14,49}$ The vehicle of the solution contains alcohol, lipophilic esters, and cyclomethicone. Pooled data from two, multicenter, randomized, parallel-group, double blind, vehicle-controlled clinical trials ${ }^{50}$ following 48 weeks of treatment showed complete cure (defined as $0 \%$ clinical involvement and mycologic cure with negative $\mathrm{KOH}$ examination and negative fungal culture) at week 52 in $18.5 \%$ of patients on efinaconazole versus $4.7 \%$ on inactive vehicle. Secondary end points were defined as mycologic cure, complete/almost complete cure $(\leq 5 \%$ clinical involvement and mycologic cure), and treatment success $(<10 \%$ clinical involvement of the target toenail), and unaffected new toenail growth. Mycologic cure was achieved in $56.3 \%$ on efinaconazole versus $16.6 \%$ with inactive vehicle. Complete or almost complete cure was obtained in $27.7 \%$ compared to $7.9 \%$ with inactive vehicle. Treatment success was seen in $47.2 \%$ compared to $18.2 \%$. Mean unaffected new nail growth was higher with efinaconazole at $5.0 \mathrm{~mm}$ in study 1 and $3.8 \mathrm{~mm}$ in study 2 compared to $1.6 \mathrm{~mm}$ and $0.9 \mathrm{~mm}$ with inactive vehicle. Thus, efinaconazole $10 \%$ solution was significantly more effective for treating DLSO than inactive vehicle alone, and efficacy rates were two to three times greater than topical ciclopirox.

\section{Comparison of safety and time requirements}

Efinaconazole has a broad spectrum of in vitro activity against dermatophytes, nondermatophytes, and yeasts. Efinaconazole 10\% solution differs from topical antifungal lacquers because it is proven to penetrate well through the nail plate into the deeper nail layers and the nail bed due to a low keratin affinity. ${ }^{51}$ Additionally, it is well tolerated with no systemic adverse effects reported in open-label studies, in both healthy volunteers and severe onychomycosis patients. ${ }^{52}$ Reported side effects include application-site dermatitis and vesicles that have been attributed to the vehicle rather than the active ingredient. ${ }^{14}$ Efinaconazole $10 \%$ solution has minimal systemic absorption. When applied to all ten toenails in severe DLSO patients and healthy volunteers, the maximum detected plasma concentration of efinaconazole and its $\mathrm{H} 3$ metabolite were negligible at 1.47 and $7.45 \mathrm{ng} / \mathrm{mL}$, respectively. ${ }^{52}$ 
Topical treatments like efinaconazole need to be applied daily for several months. Thus, patient compliance is essential to achieve good results. Efinaconazole 10\% solution needs to be brushed on the nail plate once daily for 48 weeks. In contrast to the systemic antifungals, efficacy and low toxicity come at the expense of long treatment duration and more daily time requirement. However, use of efinaconazole $10 \%$ solution is less time consuming than ciclopirox lacquer, which requires daily nail debridement and residual lacquer removal in addition to application time.

A recent study showed that use of nail polish does not reduce penetration of efinaconazole $10 \%$ solution, which is very important for female patients who do not want to show their affected nail when wearing sandals or going to the beach. ${ }^{53}$

\section{Comparison of patient satisfaction and quality of life after efinaconazole treatment}

Efinaconazole $10 \%$ solution is documented to positively impact patient satisfaction and QOL in patients with onychomycosis of the toenails. ${ }^{54}$ All aspects of QOL scores significantly improved as compared to vehicle. Additionally, improvement in QOL scores was greatest in patients who were considered clinically improved ( $\leq 10 \%$ nail involvement at week 52) and correlated inversely with percent affected nail. Therefore, any positive change in nail health may positively impact patient-perceived QOL. ${ }^{54}$

\section{Conclusion}

Though often regarded as a cosmetic problem, onychomycosis has a chronic, progressive course, with a significant burden of disease and negative impact on patient QOL. Treatments are historically limited in number and efficacy, with a high rate of relapse and recurrence. Oral antifungals are contraindicated in some cases as they carry risk of hepatotoxicity and DDIs. Disease prevalence is highest in the elderly, who are more likely to be on many medications and are at greatest risk for DDIs.

Efinaconazole $10 \%$ solution, a new triazole antifungal, was recently approved for treatment of mild to moderate DLSO of the toenails. The properties of low surface tension of the solution and low keratin affinity of the drug allow it to permeate the nail more readily and contribute to its efficacy. The mycologic and complete cure rates with efinaconazole are two- to three-fold greater than those of ciclopirox lacquer.

In addition to efficacy, efinaconazole $10 \%$ solution is safe, with little systemic absorption and remote potential for DDIs. Efinaconazole is well tolerated by the patient, and local side effects are uncommon. From the patient perspective, efinaconazole $10 \%$ solution is proven to positively impact satisfaction and QOL. Treatment of onychomycosis is important as any improvement in nail health can have a positive effect on disease burden and patient QOL.

\section{Disclosure}

Dr Tosti received honorarium from Valeant as consultant in advisory meetings. The authors report no other conflicts of interest in this work.

\section{References}

1. Gupta AK, Jain HC, Lynde CW, Macdonald P, Cooper EA, Summerbell RC. Prevalence and epidemiology of onychomycosis in patients visiting physicians' offices: a multicenter canadian survey of 15,000 patients. J Am Acad Dermatol. 2000;43(2 Pt 1):244-248.

2. Elewski BE. The effect of toenail onychomycosis on patient quality of life. Int J Dermatol. 1997;36(10):754-756.

3. Lubeck DP, Patrick DL, McNulty P, Fifer SK, Birnbaum J. Quality of life of persons with onychomycosis. Qual Life Res. 1993;2(5):341-348.

4. Schein JR, Gause D, Stier DM, Lubeck DP, Bates MM, Fisk R. Onychomycosis. Baseline results of an observational study. J Am Podiatr Med Assoc. 1997;87(11):512-519.

5. Scher RK. Onychomycosis: a significant medical disorder. J Am Acad Dermatol. 1996;35(3 Pt 2):S2-S5.

6. Milobratovic D, Jankovic S, Vukicevic J, Marinkovic J, Jankovic J, Railic Z. Quality of life in patients with toenail onychomycosis. Mycoses. 2013;56(5):543-551.

7. Roujeau JC, Sigurgeirsson B, Korting HC, Kerl H, Paul C. Chronic dermatomycoses of the foot as risk factors for acute bacterial cellulitis of the leg: a case-control study. Dermatology. 2004;209(4):301-307.

8. Bristow IR, Spruce MC. Fungal foot infection, cellulitis and diabetes: a review. Diabet Med. 2009;26(5):548-551.

9. Marty JP, Lambert J, Jackel A, Adjadj L. Treatment costs of three nail lacquers used in onychomycosis. J Dermatolog Treat. 2005;16(5-6): 299-307.

10. Arenas-Guzman R, Tosti A, Hay R, Haneke E; National Institue for Clinical Excellence. Pharmacoeconomics - an aid to better decisionmaking. J Eur Acad Dermatol Venereol. 2005;19(Suppl 1):34-39.

11. Elewski BE, Rich P, Tosti A, et al. Onchomycosis: an overview. J Drugs Dermatol. 2013;12(7):s96-s103.

12. Litz CE, Cavagnolo RZ. Polymerase chain reaction in the diagnosis of onychomycosis: a large, single-institute study. Br J Dermatol. 2010; 163(3):511-514.

13. Gupta AK, Simpson FC. New therapeutic options for onychomycosis. Expert Opin Pharmacother. 2012;13(8):1131-1142.

14. Elewski BE, Rich P, Pollak R, et al. Efinaconazole $10 \%$ solution in the treatment of toenail onychomycosis: Two phase III multicenter, randomized, double-blind studies. J Am Acad Dermatol. 2013;68(4): 600-608.

15. Loo DS. Onychomycosis in the elderly: drug treatment options. Drugs Aging. 2007;24(4):293-302.

16. Foster KW, Ghannoum MA, Elewski BE. Epidemiologic surveillance of cutaneous fungal infection in the United States from 1999 to 2002. J Am Acad Dermatol. 2004;50(5):748-752.

17. Gupta AK, Drummond-Main C, Cooper EA, Brintnell W, Piraccini BM, Tosti A. Systematic review of nondermatophyte mold onychomycosis: diagnosis, clinical types, epidemiology, and treatment. J Am Acad Dermatol. 2012;66(3):494-502.

18. del Palacio A, Cuetara MS, Garau M, Perea S. Onychomycosis: a prospective survey of prevalence and etiology in Madrid. Int J Dermatol. 2006;45(7):874-876.

19. Zaias N, Rebell G. Chronic dermatophytosis syndrome due to Trichophyton rubrum. Int J Dermatol. 1996;35(9):614-617.

20. Zaias N. Onychomycosis. Arch Dermatol. 1972;105(2):263-274. 
21. Baran R, Hay RJ, Tosti A, Haneke E. A new classification of onychomycosis. Br J Dermatol. 1998;139(4):567-571.

22. Baran R, Faergemann J, Hay RJ. Superficial white onychomycosis - a syndrome with different fungal causes and paths of infection. $J \mathrm{Am}$ Acad Dermatol. 2007;57(5):879-882.

23. Dompmartin D, Dompmartin A, Deluol AM, Grosshans E, Coulaud JP. Onychomycosis and AIDS. Clinical and laboratory findings in 62 patients. Int J Dermatol. 1990;29(5):337-339.

24. Noppakun N, Head ES. Proximal white subungual onychomycosis in a patient with acquired immune deficiency syndrome. Int J Dermatol. 1986;25(9):586-587.

25. Gupta AK, Lynch LE. Onychomycosis: review of recurrence rates, poor prognostic factors, and strategies to prevent disease recurrence. Cutis. 2004;74(1 Suppl):10-15.

26. Baran R, Sigurgeirsson B, de Berker D, et al. A multicentre, randomized, controlled study of the efficacy, safety and cost-effectiveness of a combination therapy with amorolfine nail lacquer and oral terbinafine compared with oral terbinafine alone for the treatment of onychomycosis with matrix involvement. Br J Dermatol. 2007;157(1):149-157.

27. Jennings MB, Pollak R, Harkless LB, Kianifard F, Tavakkol A. Treatment of toenail onychomycosis with oral terbinafine plus aggressive debridement: IRON-CLAD, a large, randomized, open-label, multicenter trial. J Am Podiatr Med Assoc. 2006;96(6):465-473.

28. Lahfa M, Bulai-Livideanu C, Baran R, et al. Efficacy, safety and tolerability of an optimized avulsion technique with onyster(R) (40\% urea ointment with plastic dressing) ointment compared to bifonazole-urea ointment for removal of the clinically infected nail in toenail onychomycosis: a randomized evaluator-blinded controlled study. Dermatology. 2013;226(1):5-12

29. Tosti A. Onychomycosis treatment and management. 2013. Available from: http://emedicine.medscape.com/article/1105828-treatment Accessed December 21, 2014.

30. Gupta AK. Pharmacoeconomic analysis of oral antifungal therapies used to treat dermatophyte onychomycosis of the toenails. A US analysis. Pharmacoeconomics. 1998;13(2):243-256.

31. Chang CH, Young-Xu Y, Kurth T, Orav JE, Chan AK. The safety of oral antifungal treatments for superficial dermatophytosis and onychomycosis: a meta-analysis. Am J Med. 2007;120(9):791-798.

32. Havu V, Heikkila H, Kuokkanen K, et al. A double-blind, randomized study to compare the efficacy and safety of terbinafine (Lamisil) with fluconazole (Diflucan) in the treatment of onychomycosis. BrJ Dermatol. 2000;142(1): 97-102.

33. Salo H, Pekurinen M. Cost effectiveness of oral terbinafine (Lamisil) compared with oral fluconazole (Diflucan) in the treatment of patients with toenail onychomycosis. Pharmacoeconomics. 2002;20(5):319-324.

34. de Sa DC, Lamas AP, Tosti A. Oral therapy for onychomycosis: an evidence-based review. Am J Clin Dermatol. 2014;15(1):17-36.

35. Evans EG, Sigurgeirsson B. Double blind, randomised study of continuous terbinafine compared with intermittent itraconazole in treatment of toenail onychomycosis. The LION Study Group. BMJ. 1999;318(7190):1031-1035.

36. Sigurgeirsson B, Billstein S, Rantanen T, et al. L.I.ON. Study: efficacy and tolerability of continuous terbinafine (Lamisil) compared to intermittent itraconazole in the treatment of toenail onychomycosis. Lamisil vs Itraconazole in Onychomycosis. Br J Dermatol. 1999;141(Suppl 56):5-14

37. Drake L, Babel D, Stewart DM, et al. Once-weekly fluconazole (150, 300 , or $450 \mathrm{mg}$ ) in the treatment of distal subungual onychomycosis of the fingernail. J Am Acad Dermatol. 1998;38(6 Pt 2):S87-S94.
38. Gupta AK, Lambert J. Pharmacoeconomic analysis of the new oral antifungal agents used to treat toenail onychomycosis in the USA. Int J Dermatol. 1999;38(Suppl 2):53-64.

39. Gupta AK, Fleckman P, Baran R. Ciclopirox nail lacquer topical solution $8 \%$ in the treatment of toenail onychomycosis. J Am Acad Dermatol. 2000;43(4 Suppl):S70-S80.

40. Warshaw EM. Evaluating costs for onychomycosis treatments: a practitioner's perspective. J Am Podiatr Med Assoc. 2006;96(1):38-52.

41. Elewski BE. Onychomycosis. Treatment, quality of life, and economic issues. Am J Clin Dermatol. 2000;1(1):19-26.

42. Elewski BE. Onychomycosis: pathogenesis, diagnosis, and management. Clin Microbiol Rev. 1998;11(3):415-429.

43. Gupta AK, Ryder JE, Skinner AR. Treatment of onychomycosis: pros and cons of antifungal agents. J Cutan Med Surg. 2004;8(1):25-30

44. Nolting SK, Sanchez Carazo S, De Boulle K, Lambert JR. Oral treatment schedules for onychomycosis: a study of patient preference. Int J Dermatol. 1998;37(6):454-456.

45. Hu Y, Yang LJ, Wei L, et al. [Study on the compliance and safety of the oral antifungal agents for the treatment of onychomycosis]. Zhonghua Liu Xing Bing Xue Za Zhi. 2005;26(12):988-991. Chinese.

46. Wang JL, Chang $\mathrm{CH}$, Young-Xu Y, Chan KA. Systematic review and meta-analysis of the tolerability and hepatotoxicity of antifungals in empirical and definitive therapy for invasive fungal infection. Antimicrob Agents Chemother. 2010;54(6):2409-2419.

47. Gupta AK, Ryder JE, Johnson AM. Cumulative meta-analysis of systemic antifungal agents for the treatment of onychomycosis. $\mathrm{Br} J$ Dermatol. 2004;150(3):537-544.

48. Gupta AK, Joseph WS. Ciclopirox $8 \%$ nail lacquer in the treatment of onychomycosis of the toenails in the United States. J Am Podiatr Med Assoc. 2000;90(10):495-501.

49. Tschen EH, Bucko AD, Oizumi N, Kawabata H, Olin JT, Pillai R. Efinaconazole solution in the treatment of toenail onychomycosis: a phase 2, multicenter, randomized, double-blind study. J Drugs Dermatol. 2013;12(2): 186-192.

50. Gupta AK, Elewski BE, Sugarman JL, et al. The efficacy and safety of efinaconazole $10 \%$ solution for treatment of mild to moderate onychomycosis: a pooled analysis of two phase 3 randomized trials. J Drugs Dermatol. 2014; 13(7):815-820.

51. Sugiura K, Sugimoto N, Hosaka S, et al. The low keratin affinity of efinaconazole contributes to its nail penetration and fungicidal activity in topical onychomycosis treatment. Antimicrob Agents Chemother. 2014;58(7): 3837-3842.

52. Jarratt M, Siu WJ, Yamakawa E, Kodera N, Pillai R, Smith K. Safety and pharmacokinetics of efinaconazole $10 \%$ solution in healthy volunteers and patients with severe onychomycosis. J Drugs Dermatol. 2013;12(9): 1010-1016.

53. Zeichner JA, Stein Gold L, Korotzer A. Penetration of ((14)C)efinaconazole topical solution, $10 \%$, does not appear to be influenced by nail polish. J Clin Aesthet Dermatol. 2014;7(9):34-36.

54. Tosti A, ElewskiBE. Treatment of onychomycosis with efinaconazole 10\% topical solution and quality of life. J Clin Aesthet Dermatol. 2014;7(11) $25-30$
Patient Preference and Adherence

\section{Publish your work in this journal}

Patient Preference and Adherence is an international, peer-reviewed, open access journal that focuses on the growing importance of patient preference and adherence throughout the therapeutic continuum. Patient satisfaction, acceptability, quality of life, compliance, persistence and their role in developing new therapeutic modalities and compounds to optimize

\section{Dovepress}

clinical outcomes for existing disease states are major areas of interest for the journal. This journal has been accepted for indexing on PubMed Central. The manuscript management system is completely online and includes a very quick and fair peer-review system, which is all easy to use. Visit http://www. dovepress.com/testimonials.php to read real quotes from published authors. 